\title{
Development of $\boldsymbol{P}$ - Version of General Ray Method for Parabolic Equations with Mobile Boundary Conditions
}

\author{
Armando Espíndola Pozos \\ Facultad de Ciencias Físico Matemáticas \\ Benemérita Universidad Autónoma de Puebla \\ Puebla, México \\ espinpozos@gmail.com
}

\author{
Alexandre I. Grebennikov \\ Facultad de Ciencias Físico Matemáticas \\ Benemérita Universidad Autónoma de Puebla \\ Puebla, México \\ agrebe@fcfm.buap.mx
}

\begin{abstract}
It develops the p - version of the General Ray Method (GRM) for parabolic partial differential equations for bidimensional spatial functions with compact support and mobile boundary conditions.
\end{abstract}

Keywords: Radon Transform, General Ray Method, Solution of Differential Equation.

\section{INTRODUCTION}

There are analytical and numerical methods and to solve problems with initial and boundary conditions for parabolic Partial Differential Equations (PDE), which describe thermodynamic process applied in physical areas, see [1], [2], [3], [4] and [5]. The explicit analytical method that use the Green function, in general is unknown for regions that move at the time, see [6]. The numerical methods based on Finite Difference and Finite Element at its realization leads to the solution of linear algebraic equations, requiring a lot of memory and long computer time, see [4]. For this reason it is important development and implementation of new effective numerical methods.

The $p$ - version of the GRM was developed for elliptic PDE in 2003-2007 to find the solution of boundary problems in domains of complex geometric shape, see [7] and [8]. This method is based on the use of the Direct Radon Transform (DRT) and the Inverse Radon Transform (IRT). The IRT can be quickly implemented using Fast Fourier Transform (FFT), see [9] and [10].

\section{SÍMBOLOS}

$\Omega$ :

It is a star domain in plane $\mathbb{R}^{2}$.

$\mathcal{D}\left(\mathbb{R}^{2}\right) \quad$ Space of functions defined in the plane, twice differentiable with respect to space and time variables.

\section{DEFINITIONS}

The Radon transform of some function $f \in \mathcal{D}\left(\mathbb{R}^{2}\right)$, see [11], [12], [13] and [14], is integral's family of $f$ on straight lines $l$ and these lines are determined by two parameters $p, \varphi$, where $p$ is the distance of a straight line to the origin, $\varphi$ is the angle, formed by the axis $X$ and the perpendicular segment from the origin to the line, see Fig. 1.

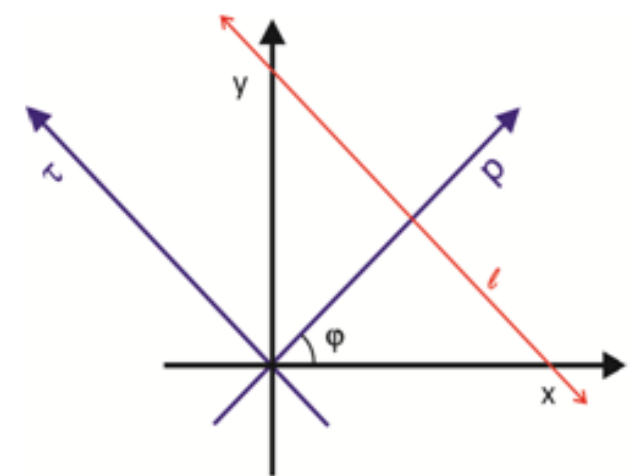

Figure 1. Straight line with its normal form $x \cos \varphi+y \operatorname{sen} \varphi=p$. 
The Radon parameterization of coordinates we will write as: $x=p \cos \varphi-\tau \operatorname{sen} \varphi$, $y=p \operatorname{sen} \varphi+\tau \cos \varphi, \tau \in \mathbb{R}$.

\section{Definition 1.}

The DRT of the function $f(x, y)$ is denoted as $R[f(x, y)]$ or $\hat{f}(p, \varphi)$ and it is defined as

$$
\hat{f}(p, \varphi)=\int_{-\infty}^{\infty} f(p \cos \varphi-\tau \operatorname{sen} \varphi, p \operatorname{sen} \varphi+\tau \cos \varphi) d \tau
$$

where $-\infty<p<\infty, \quad 0 \leq \varphi<\pi$.

Of course, the limits of integration can be finite if the function $f(x, y)=0$ out of the domain $\Omega$. Namely the DRT can be written as

$$
\hat{f}(p, \varphi)= \begin{cases}\int_{g_{1}(p)}^{g_{2}(p)} f(p \cos \varphi-\tau \operatorname{sen} \varphi, p \operatorname{sen} \varphi+\tau \cos \varphi) d \tau & ,(p, \varphi) \in \hat{\Omega}, \\ 0 & ,(p, \varphi) \notin \hat{\Omega},\end{cases}
$$

where $\hat{\Omega}=\left\{(p, \varphi): r_{1}(\varphi) \leq p \leq r_{0}(\varphi), \quad 0 \leq \varphi<\pi\right\}$ and $g_{1}(p), g_{2}(p)$, are differentiable functions on the border, see Fig. 2.

\section{Definition 2.}

The IRT of the function $\hat{f}(p, \varphi)$ is denoted as $R^{-1}[\hat{f}(p, \varphi)]$ and is it defined as

$$
f(x, y)=R^{-1}[\hat{f}(p, \varphi)]=\frac{1}{2 \pi^{2}} \int_{0}^{\pi} \int_{-\infty}^{\infty} \frac{\frac{\partial \hat{f}(x \cos \varphi+y \operatorname{sen} \varphi, \varphi)}{\partial \cos \varphi+y \operatorname{sen} \varphi-p}}{\partial p} d \varphi,
$$

where $-\infty<p<\infty$ y $0 \leq \varphi<\pi$.

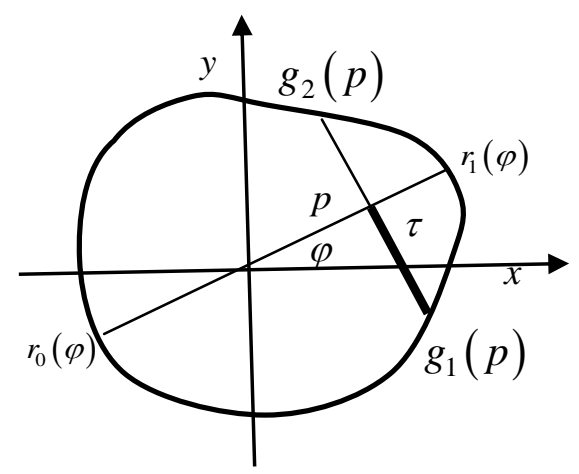

Figure 2. Domain of the functions $f$ such that $f(x, y)=0,(x, y) \notin \Omega$.

For functions twice differentiable with compact support, where the values of the first derivative at the border are equal to zeros and continuous outside the set of compact support, see [15], for DRT defined by (2) it is justified the relation

$$
R[\Delta f(x, y)]=\frac{d^{2} \hat{f}(p, \varphi)}{d p^{2}}
$$

\section{Statement of the Problem}

Solve the Dirichlet problem with border mobile for parabolic PDE in the case of spatial twodimensional functions with compact support by the $p$-version of the GRM

$$
\frac{d u}{d t}(x, y, t)-\Delta u(x, y, t)=\psi(x, y, t), \quad(x, y, t) \in \Omega(t), \quad 0<t<T,
$$




$$
\begin{gathered}
u(x, y, 0)= \begin{cases}f(x, y), & (x, y) \in \Omega(0), \\
0 & ,(x, y) \in \Omega(0),\end{cases} \\
u(x, y, t)=0,(x, y, t) \in \partial \Omega(t), 0<t<T,
\end{gathered}
$$

where $\psi, f \in \mathcal{D}\left(\mathbb{R}^{2}\right)$ are given functions, $\Omega(t)$ it is a convex bounded domain in the plane, $\Omega(t)=\left\{(x, y, t): s_{1}(t)<x<s_{2}(t), s_{3}(t)<y<s_{4}(t), \quad 0 \leq t \leq T\right.$ (fijo) $\}$, the functions $s_{1}, s_{2}, s_{3}$ y $s_{4}$ are continuous functions defined on $(0, T)$, see Fig. 3 .
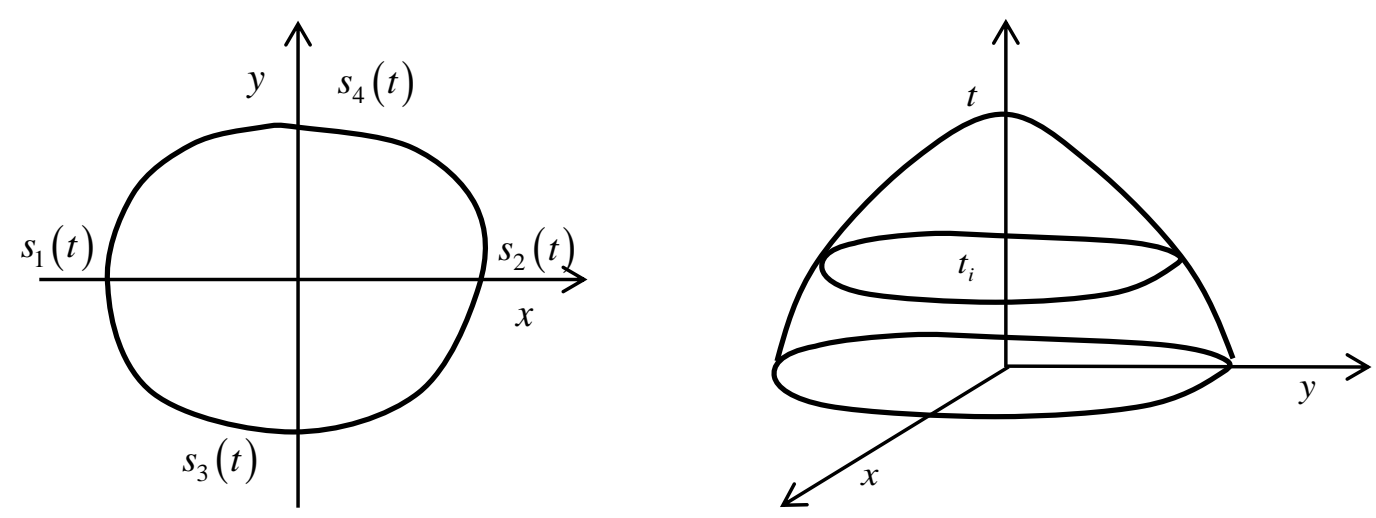

Figure 3. Domain $\Omega(t)$ with mobile border $\partial \Omega(t)$.

\section{THE SCHEME OF $\boldsymbol{P}$ - VERSION OF THE GENERAL RAY METHOD}

The scheme consists in five steps.

Step 1.

Consider the derivative of the function $u(x, y, t)$ with respect to time in Finite Differences:

$$
\frac{d u}{d t}(x, y, t) \approx \frac{u\left(x, y, t_{i}\right)-u\left(x, y, t_{i-1}\right)}{h} \operatorname{con} i=1,2, \ldots, N \text { y } t_{0}=0
$$

where $h \rightarrow 0$ it is the step size in the time, see [16].

Then the problem (5) - (7) for $t_{i}$ with $i=1,2,3, \ldots, N$, is approximated in the following way

$$
\begin{gathered}
\Delta u\left(x, y, t_{i}\right)-\frac{1}{h} u\left(x, y, t_{i}\right)=\psi_{0}\left(x, y, t_{i}\right),(x, y) \in \Omega\left(t_{i}\right), \quad 0<t_{i}<T, \\
u(x, y, 0)=\left\{\begin{array}{l}
f(x, y),(x, y) \in \Omega(0), \\
0 \quad,(x, y) \in \Omega(0),
\end{array}\right. \\
u\left(x, y, t_{i}\right)=0,\left(x, y, t_{i}\right) \in \partial \Omega\left(t_{i}\right), 0<t_{i}<T,
\end{gathered}
$$

where $\Omega\left(t_{i}\right)=\left\{\left(x, y, t_{i}\right): s_{1}\left(t_{i}\right)<x<s_{2}\left(t_{i}\right), s_{3}\left(t_{i}\right)<y<s_{4}\left(t_{i}\right), \quad 0 \leq t_{i} \leq T\right\}$,

$\psi_{0}\left(x, y, t_{i}\right)=-\psi\left(x, y, t_{i}\right)-\frac{u\left(x, y, t_{i-1}\right)}{h}$ with $t_{0}=0$.

Step 2.

Construct the family of Ordinary Differential Equations (ODE) with homogeneous boundary conditions on the variable $p$ with respect to the function $\hat{u}_{t_{i}}(p, \varphi)$, applying DRT to the equation (9). 


$$
R\left[\Delta u\left(x, y, t_{i}\right)-\frac{1}{h} u\left(x, y, t_{i}\right)\right]=R\left[\psi_{0}\left(x, y, t_{i}\right)\right],\left(x, y, t_{i}\right) \in \Omega\left(t_{i}\right), \quad 0<t_{i}<T,
$$

By the linearity of the DRT and its property (4) we have the equation

$$
\frac{d^{2} \hat{u}_{t_{i}}(p, \varphi)}{d p^{2}}-\frac{1}{h} \hat{u}_{t_{i}}(p, \varphi)=\hat{\psi}_{0 t_{i}}(p, \varphi) .
$$

Step 3.

Solve constructed ODE family for fixed angle $\varphi$ and considering the zero boundary conditions.

To simplify the notation, the step size $h$ is considered like $\frac{1}{\mathrm{~m}^{2}}, m>0$. In this way the general solution of the equation (12) is the sum of the homogeneous solution, $\hat{u}_{t_{i}, \mathrm{~h}}$, and one particular solution, $\hat{\boldsymbol{u}}_{t_{i}, p}$, see [16] and [17].

The general homogeneous solution, $\hat{u}_{t_{i}, \mathrm{~h}}$, associated with the equation (12) is:

$$
\hat{u}_{t_{i}, \mathrm{~h}}=c_{1} e^{-m p}+c_{2} e^{m p} .
$$

A particular solution, $\hat{u}_{t_{i}, p}$, of the equation (12) is:

$$
\hat{u}_{t_{i}, p}=e^{-m p} \int_{-\left(1-t_{i}\right)}^{p} \frac{\hat{\psi}_{t_{i} 0}(\xi, \varphi) e^{m \xi}}{-2 m} d \xi+e^{m p} \int_{-\left(1-t_{i}\right)}^{p} \frac{\hat{\psi}_{t_{i} 0}(\xi, \varphi) e^{-m \xi}}{2 m} d \xi .
$$

Therefore the solution of the equation (12) with homogeneous boundary conditions is

$$
\begin{aligned}
\hat{u}_{t_{i}}= & c_{1} e^{-m p}+c_{2} e^{m p}+e^{-m p} \int_{-\left(1-t_{i}\right)}^{p} \frac{\hat{\psi}_{t_{i} 0}(\xi, \varphi) e^{m \xi}}{-2 m} d \xi \\
& +e^{m p} \int_{-\left(1-t_{i}\right)}^{p} \frac{\hat{\psi}_{t_{i} 0}(\xi, \varphi) e^{-m \xi}}{2 m} d \xi,
\end{aligned}
$$

where

$$
c_{1}=\frac{e^{-2 m\left(1-t_{i}\right)} \int_{-\left(1-t_{i}\right)}^{\left(1-t_{i}\right)} \frac{\hat{\psi}_{0}(\xi, \varphi) e^{m \xi}}{-2 m} d \xi+\int_{-\left(1-t_{i}\right)}^{\left(1-t_{i}\right)} \frac{\hat{\psi}_{0}(\xi, \varphi) e^{-m \xi}}{2 m} d \xi}{\left(e^{2 m\left(1-t_{i}\right)}-e^{-2 m\left(1-t_{i}\right)}\right)}
$$

and

$$
c_{2}=-c_{1} e^{2 m\left(1-t_{i}\right)}
$$

Step 4.

It is determined the function $u\left(x, y, t_{i}\right)$ applying the inverse Radon transform to the function $\hat{u}_{t_{i}}$ obtained in the step 3.

$$
u\left(x, y, t_{i}\right)=R^{-1}\left[\hat{u}_{t_{i}}(p, \varphi)\right]
$$

Step 5.

It is obtained the approximate solution $u(x, y, t)$ of the problem (5) - (7) in a recurrent manner for $t_{0}=0, t_{1}, t_{2}, \ldots, t_{N}$.

\section{NUMERICAL EXPERIMENTS}

It is illustrated the $p$ - version of the GRM solving three Dirichlet problems with mobile border, in a bounded domain $\Omega(t)=\left\{(x, y, t): \sqrt{x^{2}+y^{2}} \leq 1-t, \quad 0 \leq t \leq 1\right\} \subset \mathbb{R}^{2} \times(0, \infty)$, using the MATLAB software. 


\section{Example 1.}

It is a synthetic example. The graph of the analytical solution is compared with the graph of numerical solution for three times, $t_{1}=0.01, t_{2}=0.05, t_{3}=0.1$, with a step size $h=0.01$ in the finite difference of the derivative with respect to time and one partition of 51 nodes in each range $[-1,1]$ where are defined the space variables, using the $p$ - version of the GRM. See Fig. 4, 5 y 6.

Solve

$$
\begin{aligned}
& \left\{\begin{aligned}
u(x, y, t)-\Delta u(x, y, t) & =-8(1+t)\left(x^{2}+y^{2}-(1-t)^{2}\right)^{3} \\
- & 48\left(x^{2}+y^{2}\right)\left(x^{2}+y^{2}-(1-t)^{2}\right)^{2}, \quad(x, y, t) \in \Omega(t), \quad 0<t<1,
\end{aligned}\right. \\
& u(x, y, 0)=\left\{\begin{array}{cl}
\left(x^{2}+y^{2}-1\right)^{4} & , \sqrt{x^{2}+y^{2}} \leq 1, \\
0 & , \sqrt{x^{2}+y^{2}}>1,
\end{array}\right. \\
& u(x, y, t)=0,(x, y, t) \in \partial \Omega(t) \quad, 0<t<1 .
\end{aligned}
$$

The exact solution is

$$
u(x, y, t)=\left\{\begin{array}{cl}
\left(x^{2}+y^{2}-(1-t)^{2}\right)^{4} & , \sqrt{x^{2}+y^{2}} \leq 1-t \\
0 & , \sqrt{x^{2}+y^{2}}>1-t .
\end{array}\right.
$$

The approximate solution constructed by $p$ - version GRM is

$$
\begin{aligned}
& u\left(x, y, t_{i}\right)=R^{-1}\left[c_{1} e^{-m p}+c_{2} e^{m p}+e^{-m p} \int_{-\left(1-t_{i}\right)}^{p} \frac{\hat{\psi}_{t_{i} 0}(\xi, \varphi) e^{m \xi}}{-2 m} d \xi+e^{m p} \int_{-\left(1-t_{i}\right)}^{p} \frac{\hat{\psi}_{t_{i} 0}(\xi, \varphi) e^{-m \xi}}{2 m} d \xi\right], \\
& \text { where } c_{1}=\frac{e^{-2 m\left(1-t_{i}\right)} \int_{-\left(1-t_{i}\right)}^{\left(1-t_{i}\right)} \frac{\hat{\psi}_{0}(\xi, \varphi) e^{m \xi}}{-2 m} d \xi+\int_{-\left(1-t_{i}\right)}^{\left(1-t_{i}\right)} \frac{\hat{\psi}_{0}(\xi, \varphi) e^{-m \xi}}{2 m} d \xi}{\left(e^{2 m\left(1-t_{i}\right)}-e^{-2 m\left(1-t_{i}\right)}\right)}, c_{2}=-c_{1} e^{2 m\left(1-t_{i}\right)} \text { and }
\end{aligned}
$$

$$
\psi\left(x, y, t_{i}\right)=8\left(1+t_{i}\right)\left(x^{2}+y^{2}-\left(1-t_{i}\right)^{2}\right)^{3}+48\left(x^{2}+y^{2}\right)\left(x^{2}+y^{2}-\left(1-t_{i}\right)^{2}\right)^{2} .
$$
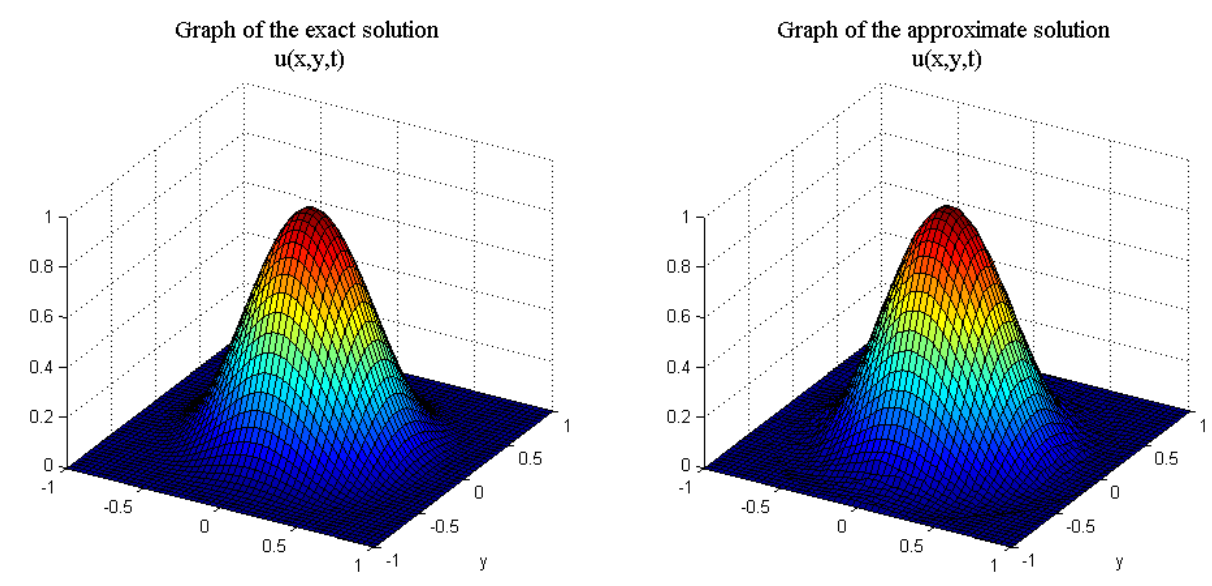

Figure 4. Graphs of the exact and approximate solution, for $t=0.01$ relative error 0.0156 , absolute error 0.0242. 

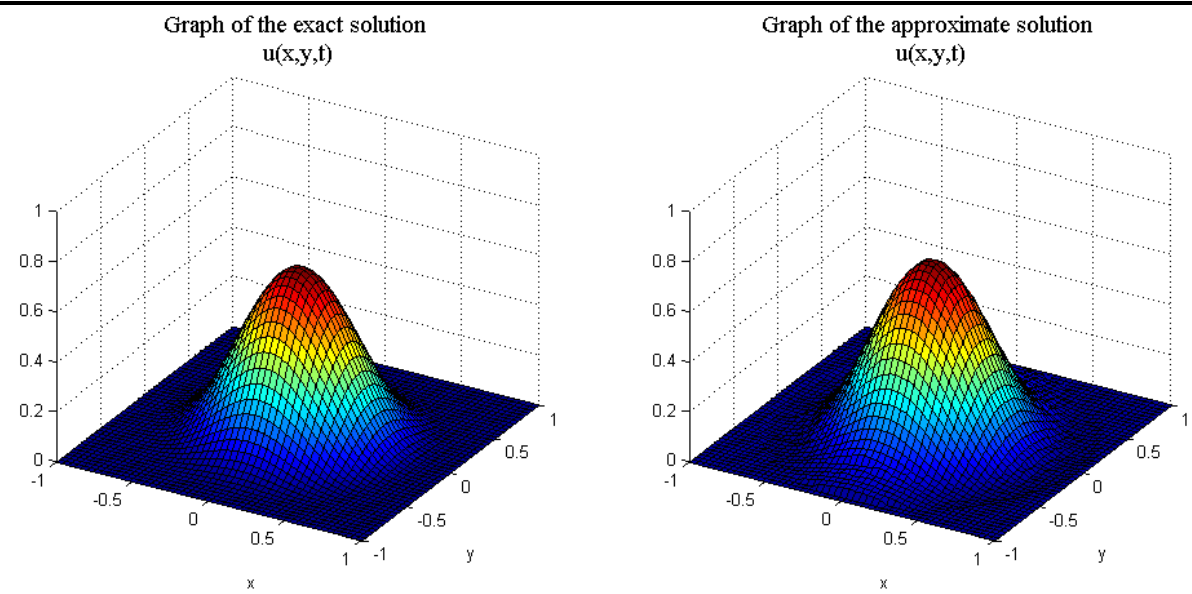

Figure 5. Graphs of the exact and approximate solution, for $t=0.05$ relative error 0.0683 , absolute error 0.0593 .
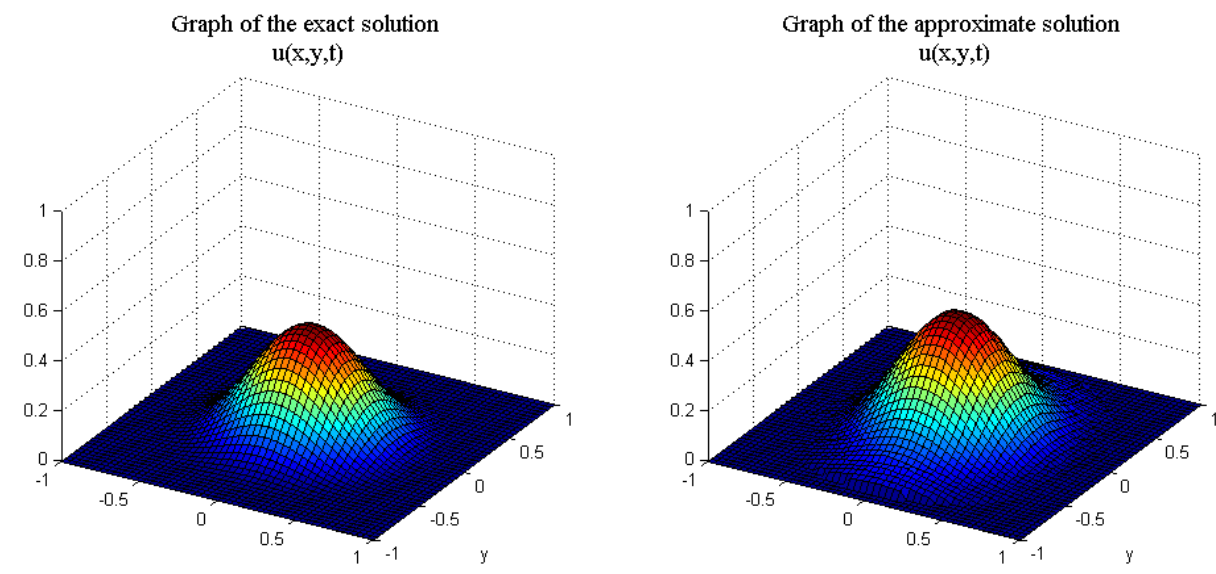

Figure 6. Graphs of the exact and approximate solution, for $t=0.1$

relative error 0.237 absolute error 0.0772 .

\section{Example 2.}

Is an example of a diffusion model of oxygen in an absorbing medium, such as tissue and in this case do not have analytic exact solution, but it was solved approximately with the Finite Element Method, see [18]. There are shown graphs of the approximated solution by the proposed method for three times, $t_{1}=0.0, t_{2}=0.05, t_{3}=0.1$, with a step size in the finite difference of the derivative with respect to time $h=0.01$ and a partition of 51 nodes in each interval $[-1,1]$ where are the spatial variables defined. See Fig. 7, 8 y 9.

Solve

$$
\begin{cases}u_{t}(x, y, t)-\Delta u(x, y, t)=-1, & (x, y, t) \in \Omega(t), 0<t<1, \\ u(x, y, 0)= \begin{cases}-\sqrt{x^{2}+y^{2}}+e^{\sqrt{x^{2}+y^{2}}-1}, & (x, y, 0) \in \Omega(0) \\ 0, & (x, y, 0) \notin \Omega(0)\end{cases} \\ u(x, y, t)=0, & (x, y, t) \in \partial \Omega(t), 0<t<1,\end{cases}
$$

Bellow we present graphs of the solution by Finite Element Method with comparing with solution by the $p$ - version of the GRM 

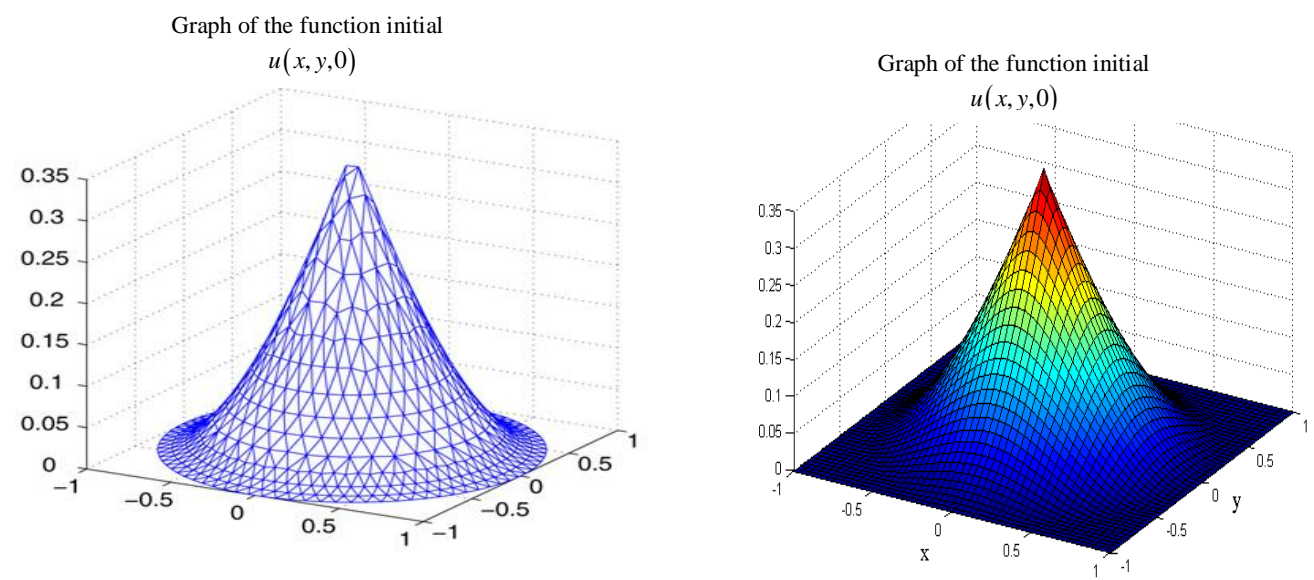

Figure 7. Graphs of the solution, for $t=0$.
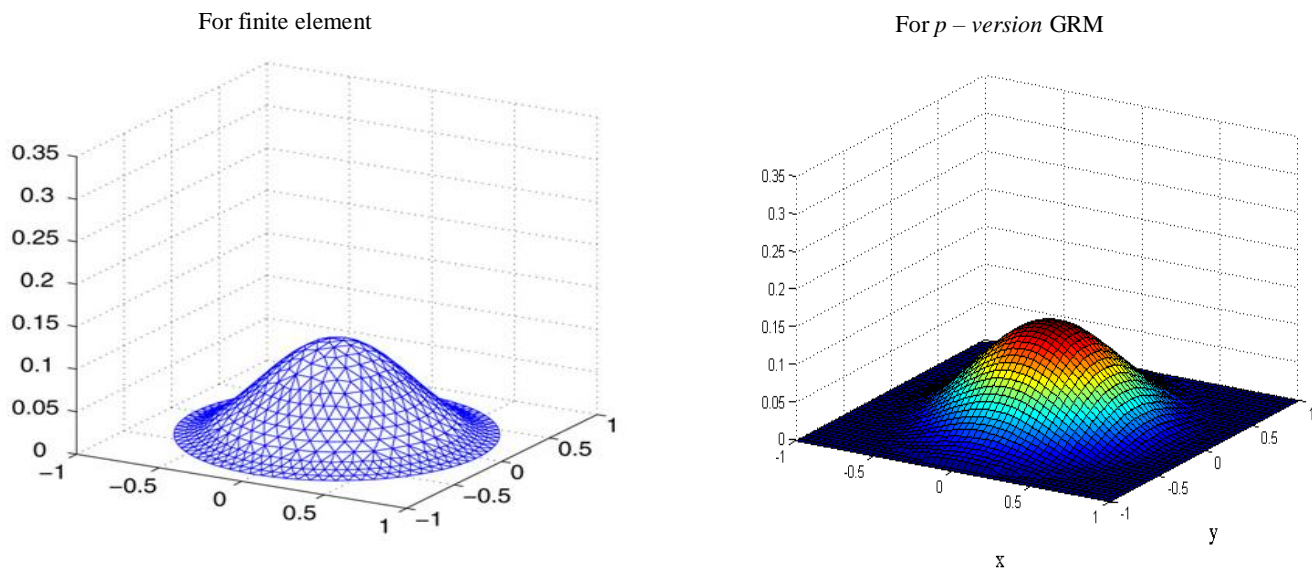

Figure 8. Graphs of the approximate solution, for $t=0.05$.
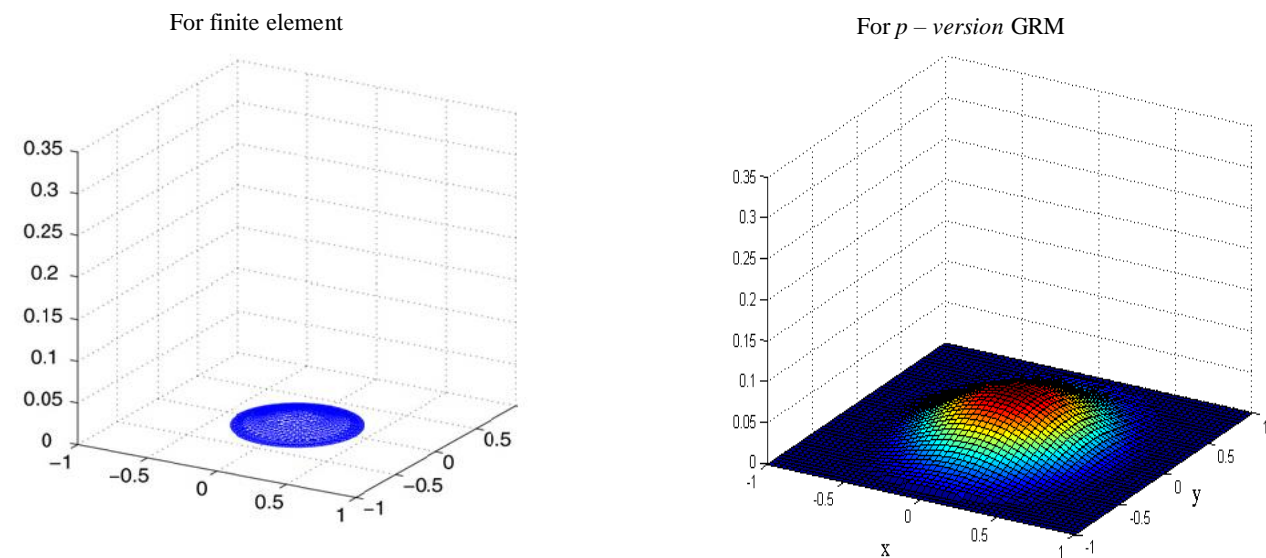

Figure 9. Graphs of the approximate solution, for $t=0.1$.

\section{Example 3.}

It is an example where the initial condition is discontinuous at the border of the circle of radius less than one, hence the considering functions have not compact support. For this example analytic exact solution is unknown. We present the graphs of the solution approximated by the proposed method for four times, $t_{0}=0.0, t_{1}=0.01, t_{2}=0.05, t_{3}=0.1$, with a step size in the finite difference of the derivative with respect to time $h=0.01$ and with 51 nodes in each interval $[-1,1]$ where are the spatial variables defined. See Fig. 10, 11, 12 and 13.

Solve 


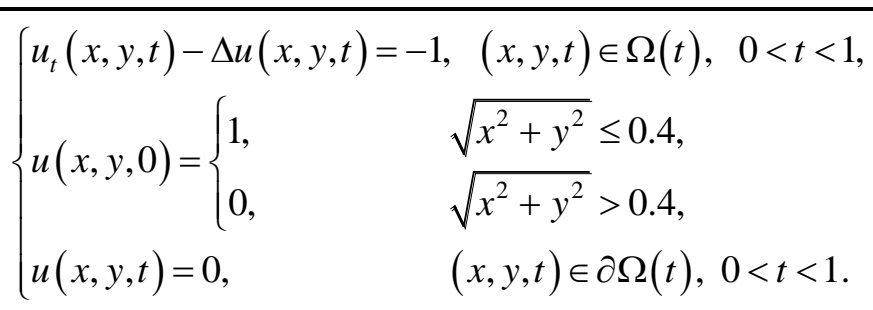

Graphs of the solution using the $p$ - version of the GRM are the next.

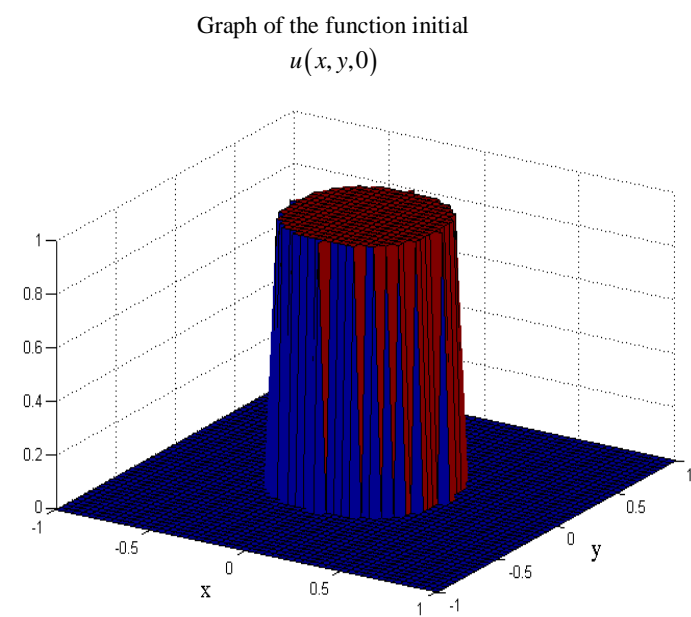

Figure 10. Graphs of the solution, for $t=0$.

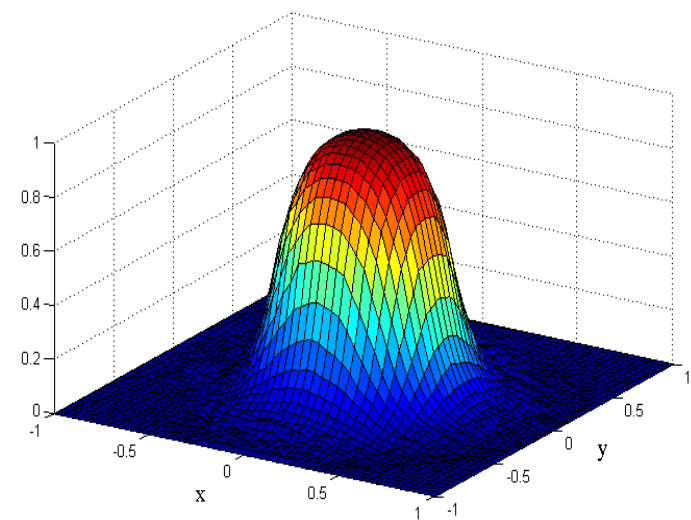

Figure 11. Graphs of the approximate solution by the $p-$ version GRM in $t=0.01$.

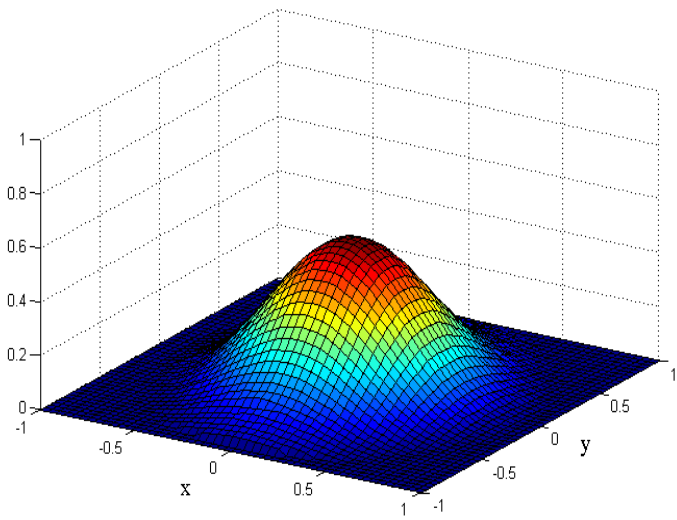

Figure 12. Graphs of the approximate solution by the $p-$ version GRM in $t=0.05$. 


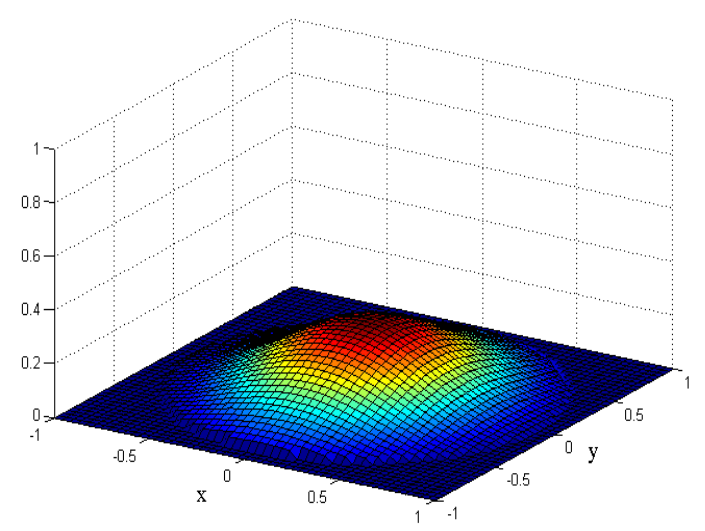

Figure 13. Graphs of the approximate solution by the $p-$ version GRM in $t=0.1$.

\section{CONCluSion}

It was developed the $p$-version of the GRM to solve numerically Dirichlet problems for parabolic PDE with mobile border for two-dimensional spatial functions with compact support and obtained an explicit numerical solution for considered problem. The results obtained in the $p$-version of the GRM are original. Numerical experiments with synthetic examples were realized in the MATLAB Software, to verify the validity of the developed $p$ - version of the General Ray Method.

\section{ACKNOWLEDGEMENTS}

Authors acknowledge to BUAP and CONACYT Mexico for their support in realization of this research.

\section{REFERENCES}

[1] Samarski A. A., Theory of Difference Schemes, Moscow (1977).

[2] Baines, M. J., Hubbard, M. E. and Jimack, P. K. A moving mesh finite element algorithm for the adaptive solution of time-dependent partial differential equations with moving boundaries. Applied Numerical Mathematics, 54:450-469 (2005).

[3] Burden Richard L., Numerical Analysis, 9 st ed., Brooks/Cole Cengage Learning (2011=.

[4] Mijailov V.P., Ecuaciones Diferenciales en Derivadas Parciales, Editorial Mir, Moscu (1982).

[5] Sanz, J. L. C., Hinkle, E. B., and Jain, A. K., Radon and Projection Transform-Based Computer Vision, Algorithms, A Pipeline Architecture, and Industrial Applications: Berlin, Germany, Springer-Verlag (1988).

[6] Tijonov, A.N. and Samarsky, A.A., Ecuaciones de Física y Matemática, Moscow: Mir, pp 492 496 (1980).

[7] Grebennikov A. I., Fast algorithm for solution of Dirichlet problem for Laplace equation. WSEAS Transaction on Computers Journal, 2(4), 1039 -1043 (2003).

[8] Grebennikov A. I., General Ray Method for Solution of Boundary Value Problems for Elliptic Partial Differential Equations, APLIEDMATH III, Memorias del Congreso Internacional en Matemáticas Aplicadas, Instituto Politécnico Nacional, México,200-209 (2007).

[9] Brigham E. Oran, The Fast Fourier Transform and its Aplications, Editorial Prentice Hall, (1988).

[10] Hwei P. Hsu Análisis de Fourier, Editorial Prentice Hall (1998).

[11] Helgason Sigurdur. The Radon Transform, Birkhauser, Boston-Besel_Berlin (1999).

[12] Jain Anil K., Fundamentals of Digital Image Processing, Editorial Prentice Hall (1989).

[13] Timothy G. Feeman, The Mathematics of Medical Imaging, Springer (2010).

[14] Stanley R. Deans, The Radon Transform and Some of its Applications, Jhon Wiley \& Sons, Inc. (1983). 
[15] Grebennikov A. I., Ana Cortés, Armando Espíndola, Radon transform of the partial derivative of a function in a bounded domain in the plane, Latin American Journal of Science, Vol. 1, no. 4 September (2014).

[16] Won Y. Yang, Applied Numerical Methods Using MATLAB, edit. Wiley-Interscience (2005).

[17] Dennis G. Zill, A First course in Differential Equations whit Modeling Applications, Tenth ed. Brooks/Cole Cengage Learning, Academic Press, Paris, 151 (1985).

[18] M.E. Hubbarda, M.J. Bainesb, P.K. Jimacka. Consistent Dirichlet boundary conditions for numerical solution of moving boundary problems. Applied Numerical Mathematics Volume 59, Issue 6, Pages 1149-1448 (2009).

\section{AUTHORS' BIOGRAPHY}

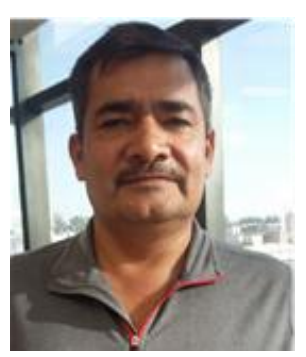

Mr. Armando Espíndola Pozos, was born in the District Federal Mexico on September 4th, 1964. His basic studies were in Puebla. He studied the undergraduate and postgraduate in Mathematics Sciences at the Mathematics College BUAP. He worked as professor at high school for 20 years. Since 2005 he is a full time research Professor at Computation Science Faculty at BUAP, Mexico. Scientific interests: Differential Equations.

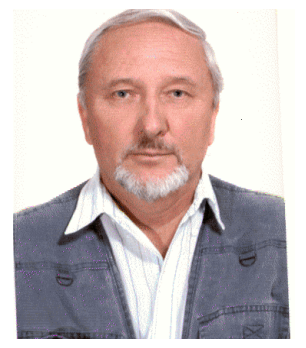

Mr. Grebennikov Alexandre, was born at 17 April 1950. Was graduated PhD at 1976 at the FCMC of Moscow State University (MSU), Publications: more than 130 articles in journals and proceedings, 3 texts of lectures, 5 monographs. He worked as assistant professor of the FCMC MSU at 1976-1989; senior staff scientist of Scientific Research Computing Center (SRCC) MSU at 1989-1999. From 1999 to present day is full professor of the Faculty of Physic and Mathematic Sciences of BUAP, Mexico. Scientific interests: splines; data processing; inverse and ill-posed problems. 